\title{
Probing the Eddies of Dancing Emergence: Complexity and Abstract Painting
}

\author{
Tara Krause \\ Atelier 1599, 6558 San Haroldo Way, Buena Park, CA 90620 USA \\ tara@tarakrause.com \\ http: //tarakrause.com
}

\begin{abstract}
Complexity and abstraction provide a fertile frontier by which to express and experience complex systems in art, with integral challenges for abstract painting: Can we create complex art without a computer? What are the simple rules by which to create that art? The author proposes a model of the artist and her materials as a cellular automaton (CA) with eight simple rules. Experiments with the New Kind of Science (NKS) Rule 1599 algorithm, abstract painting and video shorts are discussed with four observations about the plurality of CA visualization, a new abstract visual language that embraces emergence, the discovery of "eddies" of complexity within the paintings, and the resemblance of these eddies to other complex phenomena found in nature and culture. Future exploration merits investigation into the neural basis of art, and experimentation in the synaesthesic experience of complexity.
\end{abstract}

\section{Introduction}

Complexity can be generated by software/algorithmic artists and by artists using traditional techniques. This paper explores these seemingly separate paths, which are in fact convergent. Computation does not require artifice, but instead is a natural phenomena. Exciting generative art elicits an almost primal response of recognition. Complexity gives insight into processes in nature and society, and provides a basis for artistic insight.

Over the last 15 years, the concept of complexity has sparked our imagination, not just in science but in art, music and literature as well. Abstract art expert and critic F. V. O'Connor finds inspiration in the new kind of science (NKS): "Let it suffice to state flatly that there is enough dream energy and invincible charm -- enough agency in these physical invisibilities that are part of us -- to inspire a new sense of beauty, a revitalized aesthetic, and those sublime manifestations that are beyond any measurement or ethical judgment, but which can still stun us back to living life to the fullest" [1].

Much of the artistic effort on complexity has been software-based as exemplified by the work of Pegg, Trott, and Tarbell. There have been numerous art and complexity exhibitions. In the net art exhibition catalog of Abstraction Now, new media theorist Lev Manovich observed abstract and complexity as a new paradigm for software artists. 
For a painter, this presents a challenge: Without computers and software-generated algorithms, is complexity in art merely a metaphor, albeit a powerful construct? Can we move beyond complexity-inspired themes to actually create complexity? And if so, what are the simple rules that create complex art? Where is that NKS threshold of complexity where all four classes of behavior are once crossed with further embellishments having little effect?

Certainly there have been artists who have created art that can described as exhibiting NKS Classes 1 (repetitive), 2 (nested) and 3 (random) behavior. Traditional artisan craft is based on repetitive motifs. Abstract Expressionist Jackson Pollack's action paintings and the Japanese woodcuts of waves by Hokusai (1830-1840) have been shown to be fractal in nature. Medici mosaics made by the Cosmati brothers are surprising examples of NKS Class 2 patterns, as are the Byzantine mosaic from the marble pavement in Cosmedin. Earlier Chinese pottery designs appear to be examples of NKS Class 3 behavior

But what about NKS Class 4 complex behavior? This paper attempts to articulate a model in the Liebnizian spirit of the quest for the simple [2], based on Wolfram's standard of visual perception test to determine levels of complexity, and in the tradition of Salvador Dali's exhortation that "to look is to think." One is reminded of early Roman philosopher and dramatist Horace's maxim: 'Pictoribus atque poetis quidlibet audendi semper fuit aequa potestas ${ }^{l}$.' This model proposes that an artist and her materials act as a cellular automaton with simple rules to create complex art. This model is based on observations discovered in a series of both computer experiments and experimentation in the medium of acrylic polymer pigments and emulsions on canvas with a limited historical palette.

As for definitions, emergence is defined as the threshold of complexity at that coalescing moment of self-evidence when new meanings cascade into both the details and the painting as a whole. The term eddy is borrowed from physical oceanography [3] to describe the interesting details that seem to represent a small segment of adhering self-organization within the painting itself. The term primal describes that unconscious immediate response or compulsion, unedited or subjected to conscious thought. The limited palette chosen is grounded in Baroque traditional techniques, as well as pigments found in Upper Paleolithic cave art in Europe. The pigments are restricted to the ochres, siennas, umbers, black and white, with the addition of Indian yellow (traditionally made with the urine of mango leaf fed cows) and alizarin crimson or madder lake.

\section{Proposed Model}

The proposed model presents an artist and her materials acting as a cellular automaton (CA). In this sense, the art becomes an expression of this complex system. The process cannot by definition be predicted due to irreducibility. As outlined below, there are eight simple rules by which complex art can be created by this CA:

\footnotetext{
${ }^{1}$ Painters and poets alike have always had license to dare anything.
} 
1) An artist and materials act a complex system. The artist in the model does not impose a planned "cartoon" on her work in the tradition of a Caravaggisti technique, but approaches the painting as an experiment in process rather than pre-determined result. 2) The canvas is upright on the easel at a moderate temperature to exploit the reactive medium with swipes, glidings, drips and other methods of application. This allows for experimentation in the gravity-driven turbulence and flow of the materials. 3) A limited palette is used, that is grounded in human artistic tradition. Acrylic paints and polymer emulsions are set up directly on the palette, but only combined on the canvas with a palette knife or brush, not pre-mixed. 4) There is a humility of irreducibility [4]. The artist must embed the "moi" of the creative process and accept that the brain at criticality acts differently than when imposing solutions. 5) Rather than seeking to represent nature, the artist yields to a symbolic nonrepresentational (abstract) visual language of sensing complexity through the primal. The intent is to see if patterns of complex behavior can emerge. 6) The artist calibrates the painter's eye through familiarization to scientific concepts of complexity and physical processes. She learns to recognize NKS classes of behavior through repeated exposure to images of complexity. 7) The artist reaches an altered state of consciousness through the use of music and dance. She uses her whole body with motion of the lower body driving her strokes and application of painting and medium, versus standing still or sitting. 8) The artist intuitively and rigorously seeks the essence of her underlying inspiration, asking essential (simple) questions to explore the mechanism of form that moves toward transcendence as Brancusi, Modigliani, Moore, Cecily Brown and Gerhard Richter.

\section{Results and Discussion}

According to Wolfram, the presence of localized structures that move is a fundamental feature of NKS Class 4 complex behavior. Localized structures are sometimes called gliders in the Game of Life. Within class 4 CAs, systems organize themselves to where definite structures become visible. Some die out, but others persist. These persistent structures transfer information from one part of a class 4 system to another. Sometimes there are collisions between structures producing cascades of new structures.

Code 1599 is a one dimensional, 3 color (2 state) totalistic cellular automata for which no algebraic or Boolean expression exists. This simple rule works by following the rules as shown in Figure 1's rule icon. The values of the cells in an array are updated in discrete steps according to the local rule. This simple program works by having the color of each cell in successive rows be determined by the same simple rule. As a result, a pattern is produced. Interestingly, it takes 8282 steps in evolution to stabilize.

The significance of rule code 1599 is that the patterns seem to follow no definite laws. The structures emerge without anyway to predict them. One can only observe.. Wolfram uses this code to illustrate the principle of computational irreducibility and 


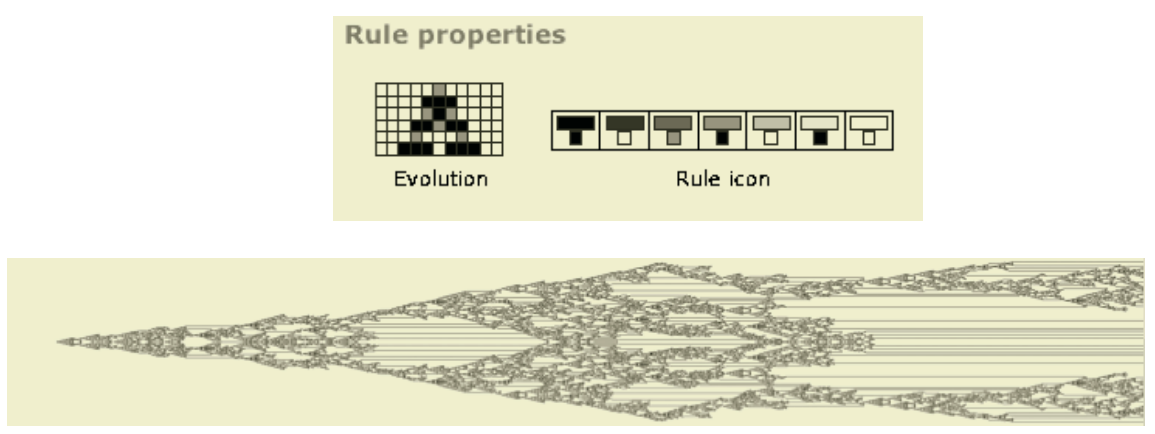

Fig. 1. (Top) NKS Rule 1599 evolution and icon rules. (Bottom) Landscape view of Code 1599 from steps 1 to 8,300 with one black cell as initial condition

its implication for the problems inherent in prediction. The only way to work out the behavior is through observation. One must perform the computation in order to see how the system behaves. This undecidability "makes it ultimately inevitable that such surprises [different forms of behavior emerging] much occur". Wolfram also observes that rule code 1599 seems to show an analog of free will in its computational irreducibility [5]. There is also a possibility that code 1599 is quasi-universal; meaning that like NKS Rule 110, it may have the potential to emulate any other system, though much more investigation is required [6].

A series of computer experiments were conducted to explore the behavior of localized structures in NKS Code 1599 during Wolfram Research Institute's NKS Summer School 2004 at Brown University. An automated search of specified initial conditions of range 1 to 3,000,000 run to step 300 yielded 20,416 files that were sorted into four separate categories of behavior: Dead within 300 steps (11\%), Single chain of "lady bug" like structures (19.2\%), Single chain of "railroad track" like structures (2\%) and those other initial conditions that might create interesting patterns $(67.7 \%)$. The last category of 13,820 images was visually analyzed. From this analysis, it was observed that the behavior varies across conditions, with only 2 equivalent initial conditions creating the 8,282 steps of evolution. There appeared to be definite structures, some of which were resistant to varying specified initial conditions, and evident patterns of smaller subcomponent elements in both specified and random initial conditions [7].

\subsection{Observation 1: There Are a Myriad of Ways to Express Cellular Automata}

The experiments were followed by an investigation into visualizing the onedimensional CA 1599 in three dimensions. These visualizations included various coloration schemes, as well as perspectives such as spinning radius, beaded hanging, and birds-eye view. While interesting, none of these approached the beauty of the CA in the original first dimension.

This raised new questions in terms of the physicality of painting in 2 dimensions. If all the visualizations represented unique but acceptable views, could there not be 
other possibilities in expressing the complexity? This opened the degrees of freedom, aptly expressed by Todd Rowland: "Some people describe 1599 mathematically in terms of 1s and 0s. Other people call it cool babushka, and still others teach their children to dance to it" [8]. Moreover, like the Modernist artists struggling with expressing the fourth dimension, contemporary artists are confronted with the phenomena of the first dimension of the computational universe.

There are a myriad of ways of expressing a CA. Stephen Wolfram's use of the cone shell (conus textile) is the "classical" example of a 3 dimensional expression of a CA. The cone shell is a 3-D coiled object with the surface as a 2-D pattern generated by a 1-D CA at the living edge of the organism. Another example developed in the experimentation was a tapestry [9]. Birthed of Flatland [10], the tapestry can be conceived as a cellular automata causal network. The digital art image, NKS_Nasij (2003), was based upon fragment of a woven golden tapestry (c. 1302) for the emperor Ghazan Khan, grandson of Ghenghis Khan, in Tabriz with Code 1599 superimposed [11]. Considering the cultural complexity of this tapestry's creation and use, this might be an NKS class 4 localized structure that maintains its coherence while moving through its cellular space.

\subsection{Observation 2: A New Abstract Visual Vocabulary Embraces Emergence}

Yet even with these various visualizations and computer-generated expressions, it was still not evident that emergence as a result of process was possible in painting. Experimentation in the medium of acrylic polymer on canvas was necessary. Earlier efforts in printmaking with experiments in 2003 demonstrated that monotype printing as a reactive medium might produce some evidence of complexity; but due to the medium's heavy reliance on fluid flows of turpentine and inks, it was not conclusive enough.

The acrylic painting experiments used simple rules as outlined in section 2 of this paper for the total of 18 paintings in the Dancing Emergence series by Krause. The paintings ranged from 30 inches by 40 inches, to 18 inches to 12 inches. The surprise of the experimentation was that a new abstract visual vocabulary developed. Emergence became an artistic process.

However, there remained the challenge of scaling up the size of the canvas while still maintaining the capacity for emergent patterns. This Richard Serra scale intent became: Can one evoke a complex reaction from the viewer using these images projected on a large screen? How can one create a Rothko-like chapel of a procession of images that evoke complexity?

To work around this challenge, a short film was made that combined Katarina Miljkovic's music compositions of different CA rules (Rules 41 and 1599 along with a Turing Machine) with video footage of the paintings. This experiment demonstrated that this new language was rich enough for further experimentation. Following that up, the Dancing Emergence paintings were filmed close-up in motion synchronized to music. In editing the footage, it was discovered that while the paintings cohered as 
complex, there were also many individual close-up details of complex "eddies" or localized structures.

\subsection{Observation 3: The Eddies Stand on Their Own Terms as Local Structures of Complexity Within the Larger Work}

In the approximately 50 minutes of that raw video footage, there were at least 174 examples of images that can be described as complex eddies. Figure 2 below shows the visual evidence of four of these particular eddies. These are still frames of actual details of the larger acrylic polymer and emulsion paintings on canvas.

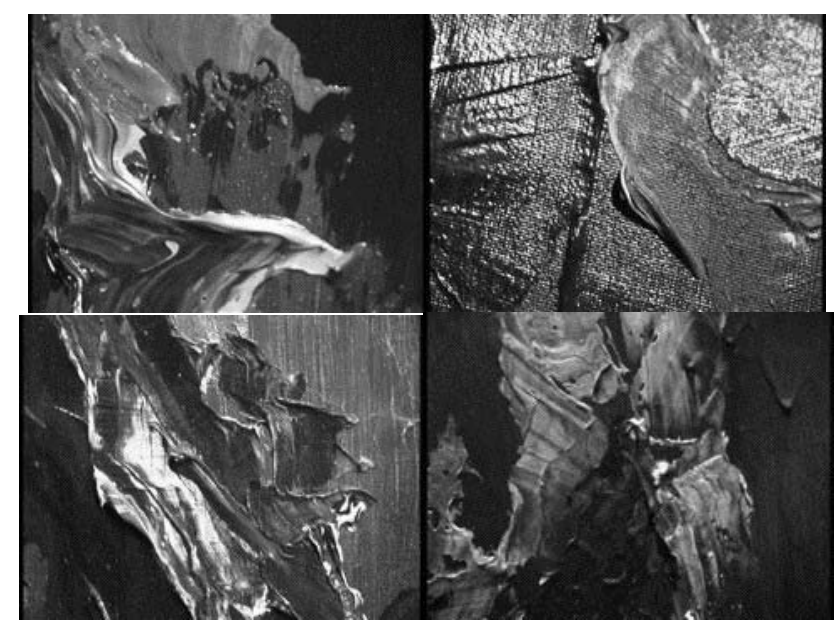

Fig. 2. Details of acrylic polymer and emulsion on canvas. Video stills from Dancing_Emergence painting series with the following titles of (clockwise) $D E \_17, D E \_19, D E \_12$, and $D E \_15$ (2004)

\subsection{Observation 4: The Complexity in the Abstract Paintings Resemble Other Complex Phenomena in Culture}

The observation of the paintings' eddies sparked a recognition of similar complex patterns in some Upper Paleolithic microliths. Photographs of Paleolithic rock art seem to appear to show a web of class 4-like finger fluting (intentional engravings) over the initial images. Examples of such are the yellow stallion in the Apse of the Cave of Lascaux (c. 17,000 years ago in the Magdalenian era); another Magdalenian period carved image from the Bases-Pyrénées in France; and the finger tracings of the Gargas and Cosquer caves. One class of Paleolithic finger fluting has been theorized to result from lower body motion. The phenomena of finger flutings in the Rouffignac Cave were to have resulted from moving from the hips with bending, twisting and shifting weight on feet [12]. Some experts have also observed that the size of the handprint signatures in more than twenty caves throughout Italy, France and Spain 
point to women shamans and that this indicated a female shamanic role in the spiritual and creative life of the Paleolithic clans.

Even more provocative are the theories of South African San rock art expert Davis Lewis-Williams [13]. His shamanistic art neuropsychological model holds that shamans created these microlithic abstract images from trance phosphenes or entopics (seen by the eye when eyelids are shut) while in an altered state of consciousness (ASC) mode. In Lascaux cave art, Mario Ruspoli stressed that these images have to seen as a whole and mused that perhaps in the flickering of the fire to the rhythm of drums, a shaman in the Lascaux engraved the figures as he told the story before his initiates, that the movements of his hand and the act of drawing combined in its meaning.

Another example of complexity expressed in culture is the Taoist Neo-Confucian taxonomy of $L i$ patterns that represent dynamic forms found in nature, considered as laws or principles and expounded on by Chu Hsi during the Sung dynasty (960-1279) and Ch'en Shun. Many of David Wade's categories of $l i$, such as breccia and fracture, are strikingly NKSesque if not Class 4 in appearance [14].

These observations raise more questions than answers. What is the neural basis of the artistic creation and perception of such complexity? Is there an interconnection with neural laws of art as theorized by neuroscientist V.S. Ramachandran, such as peak shift, grouping, contrast and isolation [15]? This frontier expands beyond the realm of vision when the question is asked: How does one express and experience cellular automata synaesthesically, where all senses are integrated?

More investigation should be done to address the complexity science underneath these observations: Are these observations a representation of natural processes or a result of a complex process? What is the role of irreducibility? Is the surprise of emergence a result of the process? It does not seem that this could be achieved through an imposed Caravaggisti method. The artist and her materials become the cellular automata.

\section{Conclusion}

As observed, the creative use of cellular automata provides fertile ground by which complex systems can be expressed and experienced in art, particularly in abstract painting. The artist and her materials function as a computational machine, or cellular automata to create complex art out of simple rules. The patterns in paintings resulting from this CA may resemble complex phenomena in some natural as well as cultural processes. Like the Modernist artists who struggled with expressing Einstein's fourth dimension, contemporary artists are challenged with the phenomena of complexity. New artistic insights from complexity and cellular automata can spark our imaginations, with the songs of the past sung with the rituals of the present to weave the vision and language of the future, where we can sense the kernel of our humanity and environment in the story space of the universe, and where gliders and persistent structures can lead us to transcendence. 


\section{Acknowledgements}

I would like acknowledge: Drs. Jiri Kroc; composer Katarina Miljkovic of New England Conservatory of Music; Stephen Wolfram and the Wolfram Research Institute team, particularly Todd Rowland, Catherine Boucher, Jason Cawley and Ed Pegg Jr.; and Dale C. Krause of the Marine Science Institute of UCSB.

\section{References}

1. F.V. O'Connor: Commentary No.2 (2004) http://members.aol.com/FVOC/comment.html

2. G. Chaitin: Irreducible Complexity in Mathematics (2004) http://www.cz.auckland.ac.nz/CDMTCS/chaitin/latest.html

3. D.C. Krause: The Self-Organized Marine Biological System. In: Soto, L.A, (ed): AyalaCastanares. Sciencia del Mar y Lmnol. Univ. el NAL. Auton. Mexico (2003)

4. Private communication: J. Cawley, T.Rowland and K. Miljkovic, NKSSS2004 (2004)

5. S.Wolfram: A New Kind of Science. Stephen Wolfram, Champaign (2002)

6. Private Communication: S. Wolfram during NKSSS2004 (2004)

7. T. Krause: Greeting the Muse, NKS Code 1599: Behavior, Motifs \& Potentialities for an NKS Way of Art. NKS Summer School 2004. Boston (2004)

8. Private communication: T. Rowland of WRI (2004)

9. Private communication: J. Cawley, T. Roland, R. Philips of WRI and K. Miljkovic of New England Conservatory of Music during NKSSS2004 (2004)

10. E.A. Abbott: A Romance of Many Dimensions (1884)

11. T.Krause: Cellular Automata, Undulating Jellies \& Pulsing Bonita. Video, NKS2003, Boston (2003). Color images can be found at http://tarakrause.com

12. K. Sharpe and L. van Gelder. Finger Flutings in Rouffignac Cave, France. (2003)

13. D. Lewis-Williams: Mind in the Cave: Consciousness and the Origins of Arts. WW Norton (2004)

14. D. Wade: Li: Dynamic Form in Nature. Wooden Books, New York (2003)

15. V.S. Ramachandran: A Brief Tour of Human Consciousness. Pi Press, New York (2004) 